\title{
Intracranial Neurostimulation for Epilepsy
}

\author{
A. Parrent
}

Epilepsy affects about $1 \%$ of the population, and it is estimated that $5-10 \%$ of patients with epilepsy will become medically intractable. Of this group, less than half are considered good candidates for conventional resective epilepsy surgery. In the remainder we are continually seeking novel methods for treating seizures.

\section{Rationale}

Interest in deep brain stimulation (DBS) was rekindled in the late 1980's when Benabid proposed DBS for the treatment of tremor $^{1}$. In the movement disorder world DBS has been used for the treatment of tremor, Parkinson's disease and dystonia. Its application has recently expanded into the neuropsychiatric realm for the treatment of depression, obsessive-compulsive disorder, Tourette's syndrome and other conditions.

Although the precise mechanism by which DBS has its effects is not known, it appears empirically that high frequency stimulation has the effect of functionally and reversibly suppressing the function of deep brain structures while low frequency stimulation has the opposite effect.

Epilepsy can be thought as arising from an imbalance between excitatory and inhibitory processes in the brain leading to uncontrolled electrical discharge - either focally or generalized. Intracranial neurostimulation for epilepsy uses one of the following strategies:

1. Reduction of seizure threshold or cortical excitability.

2. Direct stimulation of, and suppression of a seizure focus.

\section{Cerebellar Stimulation}

Cooper ${ }^{2}$ proposed stimulation of the anteromedial cerebellar cortex in the early 1970s based on experimental evidence that stimulation of the cerebellum could improve generalized seizure activity and focal limbic seizures in some animal models ${ }^{3}$. The results of the various published articles dealing with cerebellar stimulation in humans are hard to interpret given the differing patient selection criteria, treatment protocols and outcome measurements. Cooper reported seizure reduction of at least $50 \%$ in 18 of 34 patients. Krauss and Koubeissi ${ }^{4}$ summarized data from 11 uncontrolled studies involving 115 patients, demonstrating improvement in 87 (76\%). In contrast, two double blind, controlled studies involving 17 patients failed to show significant seizure reduction during chronic cerebellar stimulation $^{5,6}$. Van Buren's study has been criticized for the small number of patients, incorrect calculation of seizure reduction $^{7}$, and a stimulation protocol which, in Cooper's opinion promoted rebound seizures. Cooper ${ }^{8}$ also criticized the lack of biocalibration of the stimulator settings suggesting that subtherapeutic stimulation could augment rather than inhibit seizure activity. Velasco et $\mathrm{al}^{9}$, in their randomized double blind study involving five subjects, found that generalized tonic clonic seizures were significantly improved in the treatment group by the third double blind month, and in all patients during the open label portion of the study. They suggested that there was a delayed and progressive effect of cerebellar stimulation on generalized tonic clonic seizures and tonic seizures. Davis and Emmonds $^{7}$ and Bidzinski et $\mathrm{al}^{10}$ have argued for a carry-over effect after as little as 10-12 days of temporary stimulation.

Generally, cerebellar stimulation is in the $10-20 \mathrm{~Hz}$ frequency range, intermittently on and off for one to eight minutes. Davis ${ }^{11}$ felt that stimulation amplitude should be adjusted to deliver a charge density of $1-4 \mu \mathrm{C} / \mathrm{cm}^{2} /$ phase.

There are very little solid data to support the use of cerebellar stimulation for the treatment of epilepsy, and enthusiasm for cerebellar stimulation has waned with the exception of a few centers where it is primarily used for the treatment of spasticity in cerebral palsy ${ }^{11}$. Although it is unlikely to occur, a double blind randomized study of cerebellar stimulation enrolling large numbers of patients and appropriately stratified for seizure type is probably warranted.

\section{Caudate Nucleus Stimulation}

A number of studies suggest that the caudate nucleus is part of an inhibitory system capable of suppressing seizure activity. La Grutta found that stimulation of the caudate nucleus inhibited hippocampal and amygdalar as well as temporal neocortical epileptic activity in stimulation-induced and focal penicillin models of epilepsy in the cat ${ }^{12-14}$. Caudate stimulation has also been shown to decrease seizure activity in a cobalt model of neocortical epilepsy in the $\mathrm{cat}^{15}$. In this study, caudate stimulation was more effective for seizure foci in the anterior forebrain than posterior cortex. Sorbera et $\mathrm{al}^{16}$ proposed a cortical-subcortical loop from caudate to substantia nigra then amygdala as a mechanism for the effects of caudate stimulation on temporal limbic seizures.

In humans, a number of case series have reported benefit from caudate stimulation. In 41 of 57 patients subjected to a 20 day period of trial stimulation of the caudate head, Chkhenkeli et $\mathrm{al}^{17,18}$ found that high frequency stimulation $(30-100 \mathrm{~Hz})$ elicited the appearance of, or enhanced preexisting epileptiform discharges, whereas low frequency stimulation $(4-8 \mathrm{~Hz})$ reduced interictal spikes and aborted epileptiform discharges in the mesiobasal temporal lobe. Bilateral suppressive effect was seen

From the Epilepsy Program, Western University, University Hospital, London, Ontario, Canada.

Received January 26, 2012. Final Revisions Submitted August 7, 2012. Correspondence to: A. Parrent, Epilepsy Programme, Western University, London Health Sciences Centre, 339 Windermere Rd, London, Ontario, N6A5A5, Canada. 
with unilateral stimulation, and the effect was seen with stimulation of the ventral but not the dorsal caudate head. The effect outlasted the duration of stimulation and the duration of benefit increased over the period of the study.

Sramka reported benefit in two of eight patients undergoing chronic caudate stimulation utilizing variable stimulation parameters ${ }^{19}$. Chkhenkeli et $\mathrm{al}^{17}$ used chronic low frequency stimulation and reported that 9 of 18 patients were seizure free, with no apparent specificity with regard to seizure type. They noted that the decrease in frequency and severity of clinical seizures developed gradually over the course of the study becoming much more evident three to four weeks into the treatment. They hypothesized that this phenomenon, analogous to Rasmussen's "running down" phenomenon ${ }^{20}$ was a result of changes developing in a dynamic epileptic system under the influence of therapeutic brain stimulation.

No controlled studies of caudate stimulation in humans have been reported.

\section{Thalamic Deep Brain Stimulation}

The nonspecific thalamic nuclei, with their diffuse projections to wide cortical areas include the reticular nuclei, the anterior nuclei and the intralaminar nuclei ${ }^{21}$. Some of these nuclei are organized as a network known as the reticulothalamocortical system ${ }^{22}$ that connects the thalamus to the cortex. This circuit has been implicated in the regulation of the cortical excitability, in the generation of the cerebral rhythms, in the control of consciousness and in the origin of generalized seizures.

There is evidence that certain generalized seizures originate with the thalamus and its connections with the cortex. Thalamic electrical stimulation in cats produces EEG patterns similar to those seen in typical absence seizures ${ }^{23}$. Moreover, thalamic recordings patients with absence epilepsy demonstrate three-persecond EEG discharges during typical seizures ${ }^{24}$. Gloor proposed the hypothesis that the spike and wave discharges (SWDs) are produced by increased responses of cortical neurons to the thalamocortical volleys that normally produce sleep spindles but are altered in absence epilepsy ${ }^{25}$.

The centromedian and anterior nuclei of the thalamus have been used as stimulation targets for the treatment of epilepsy.

\section{Centromedian (CM) Stimulation}

The CM nucleus of the thalamus has been used because it is a large structure, a good stereotactic target and an intralaminar nucleus, part of the reticulothalamocortical system. High frequency stimulation of this nucleus in experimental animals causes cortical desynchronization ${ }^{26,27}$ and blocks epileptic discharges ${ }^{28}$.

Centromedian stimulation for epilepsy treatment in humans was first reported by Velasco et al in $1987^{29}$, and they have published numerous subsequent papers on the topic. They believe that $\mathrm{CM}$ stimulation is most effective for generalized tonic-clonic and atypical absence seizures in the setting of the Lennox-Gastaut syndrome, reporting an overall $81 \%$ seizure reduction in this group ${ }^{30,31}$. There is a concomitant reduction in generalized slow spike-wave complexes, focal frontal spikes and secondarily generalized EEG discharges ${ }^{32}$. In contrast, CM stimulation is not effective in the management of complex partial seizures and focal spikes in the temporal regions.

Velasco thought that predictors for good outcome included precise selection of patients and accuracy in the localization of the electrodes. They advocated ventriculographic guidance and electrophysiologic confirmation of electrode localization. The optimal target is considered to be the basolateral portion of the $\mathrm{CM}$ nucleus. Patients with recruiting responses in response to low frequency stimulation $(6 \mathrm{~Hz})$ and EEG desynchronization and negative direct current (DC) shifts generated by high frequency stimulation were considered to have adequate electrophysiological confirmation. The anticonvulsant effect of $\mathrm{CM}$ stimulation appears to persist for some months after discontinuing CM stimulation.

Fisher conducted the only placebo-controlled double-blind study to assess the efficacy of CM stimulation ${ }^{33}$. This small study involved seven patients, and used a cross-over design in which patients underwent an initial three month period of stimulation or placebo, three months of "washout" with the stimulator off, and three months of treatment opposite to that in the initial three months. One of the patients was dropped from the protocol for compassionate reasons when the initial randomized treatment period produced substantial seizure reduction. $\mathrm{n}$ the remaining six patients there was an overall $30 \%$ reduction of generalized seizures when the stimulator was on, and an $8 \%$ reduction when the stimulator was off compared to baseline. This difference was not statistically significant.

The major differences between the Fisher group and the Velasco group were the method of CM localization and study $\operatorname{design}^{21,30,33}$. Velasco emphasized the importance of stimulation induced recruiting responses at low frequency, and EEG desynchronization at high frequency as a physiological index of correct electrode placement. Fisher's group used anatomical stereotactic methods based on identification of the anterior and posterior commissures. They did not obtain recruiting responses in any of their patients.

Velasco et $\mathrm{al}^{31}$ subsequently reported a double-blind crossover study in 13 patients. They found that seizures were significantly reduced during the stimulation-on and stimulationoff phases of the study compared to baseline. However, there was no significant difference in seizures between the 'on' and 'off' periods of the study. There were two patients who were explanted and experienced seizure recurrence to baseline levels at four and six months. This led them to conclude that CM stimulation has a residual effect on seizure occurrence that outlasts the duration of stimulation by as long as three or four months.

At present there is sufficient evidence to say that chronic CM stimulation is safe. Conclusive evidence of efficacy will require a double-blind placebo-controlled study that addresses the issues of physiological localization of the target in the CM nucleus as well as the potential long lasting effect of stimulation.

\section{Anterior Nucleus Stimulation}

The anterior nucleus (AN) of the thalamus as a part of the limbic system has important connections with cortical and subcortical structures. AN receives input from cingulate cortex, the hippocampus via the fornix, and the mammillary bodies (MB) via the mammillothalamic tracts (MT). The major AN 
outputs are directed to the hippocampus and cingulate cortex. These structures are organized as the well-known circuit of Papez: hippocampal formation - MB - AN - cingulate cortex hippocampal formation. Because of this strategic position, AN may mediate cortical and subcortical interactions.

Animal studies have demonstrated the involvement of AN in the generation of pentylenetetrazol (PTZ) induced seizures ${ }^{34-36}$. Pharmacological inhibition of AN, lesioning of the mammillothalamic tract or mammillary bodies ${ }^{37,38}$, and high frequency stimulation of $\mathrm{AN}^{35,39}$ all abort or attenuate the development of PTZ-induced seizures. AN lesioning or high frequency stimulation are also anticonvulsant in the pilocarpine model of epilepsy in the rat ${ }^{39,40}$.

In the early 1980's Cooper stimulated the AN in six patients with complex partial seizures and no localizable focus ${ }^{41}$. Seizure frequency improved by more than $60 \%$ in five patients, and medications were reduced by $30 \%$ in the group. In 1988, Sussman reported the results of AN stimulation in five patients ${ }^{42}$. There was an improvement in seizure activity in three patients. Two of four patients with complex partial seizures were improved. A fifth with secondary generalized epilepsy experienced complete cessation of convulsions and drop attacks but persistence of complex partial seizures and absences.

Hodaie, Andrade and their group ${ }^{43,44}$ found that implantation of bilateral AN electrodes reduced seizure frequency by more than $50 \%$ in five of six patients. Activation of the stimulators produced no further seizure improvement, raising the possibility that the benefit was due to a microthalamotomy effect.

A double-blind randomized multicenter study of AN stimulation has been carried out ${ }^{45}$. One hundred and ten patients were implanted with bilateral AN electrodes. One month postimplantation, they were randomized to stimulation vs no stimulation for a period of three months. This was followed by a period of unblinded stimulation. At the end of the blinded phase, the stimulation group saw a $40.4 \%$ reduction of seizures compared to $14.5 \%$ in the control group. The differences in seizures frequency between active and control group were significant for seizures originating from one or both temporal lobes but not for seizures originating from frontal, parietal or occipital lobe. With long-term follow-up there was a $41 \%$ decrease in seizure frequency at 13 months and $56 \%$ decrease at 25 months.

The current data suggest that AN stimulation is safe and can reduce seizure frequency in some patients. The effect of AN stimulation may improve over time. It may be more effective for patients with temporal lobe foci. Results suggest that this procedure may have a palliative role in some patients with medically refractory epilepsy. Further studies may help clarify optimal patient selection.

\section{Subthalamic Nucleus Stimulation}

Since Iadorola and Gale first described the nigral control of epilepsy system in 1982, other studies have confirmed a modulatory role of the basal ganglia and related structures on certain types of seizures ${ }^{46-53}$. Direct inhibition of the STN with GABA agonists has anticonvulsant effects on amygdala kindled seizures in the $\mathrm{rat}^{54}$ and suppresses SWDs in a rat model of absence epilepsy ${ }^{55}$. There are a number of animal models in which subthalamic nucleus (STN) stimulation reduces seizure frequency. High frequency STN stimulation suppresses spikewave discharges and seizures in the GAERS model ${ }^{56}$, prevents seizure generalization in a rat kainic acid model $^{57}$ and reduces clonic seizures in the fluoroethyl acute seizure model ${ }^{58}$.

The human studies of STN stimulation for epilepsy come from two groups. In 1998, Benabid and his group in Grenoble carried out their first case of STN stimulation in a patient with cortical dysplasia and intractable seizures ${ }^{59}$. A total of five patients underwent STN stimulation at their center ${ }^{60,61}$. Three patients clearly responded to the treatment with seizure reductions of $71-84 \%$. Bilateral stimulation was felt to be more effective than unilateral stimulation, using stimulation parameters similar to those used in patients with movement disorders (130 Hz, 60-90 mcsec). The Cleveland Clinic reported on four patients with STN stimulators demonstrating benefit in two patients with $42 \%$ and $75 \%$ reduction of seizure frequency along with reduction of seizure severity and duration ${ }^{62}$. Continuous and intermittent stimulation appeared to be equally effective.

The issues of STN stimulation for epilepsy are similar to those of other deep brain sites. The studies are all uncontrolled case series that serve to demonstrate the safety of the technique and the potential for benefit. Controlled studies will be required in larger numbers of patients in order to delineate its role in the treatment of epilepsy. The STIMEP trial (Assessment of Subthalamic Nucleus Stimulation in Drug Resistant Epilepsy) was a randomized, double-blind controlled clinical trial designed to assess the role of STN stimulation for seizure control. It was terminated due to poor enrollment (http://clinicaltrials.gov/ct2/ show/NCT00228371).

\section{Stimulation of the Epileptic Focus \\ Hippocampal Stimulation}

Hippocampal stimulation would be an appealing option for the treatment of patients with mesial temporal lobe epilepsy (TLE) for whom resective surgery is not possible or would pose significant functional risks. This would include patients with bilateral mesial TLE, or patients with unilateral mesial TLE involving a dominant hippocampus that is essential for memory function.

Velasco carried out some of the early studies of hippocampal stimulation in humans ${ }^{63,64}$. In ten patients who had undergone implantation of subdural or depth electrodes in the temporal region for investigation prior to temporal lobectomy, bipolar high frequency $(130 \mathrm{~Hz})$ stimulation was applied continuously for two to three weeks. In seven patients, complex partial and secondarily generalized seizures were abolished after day 6 of stimulation and interictal spikes were either eliminated or substantially reduced. The best results were obtained from depth electrode contacts located within the pes hippocampus near the amygdala, or subdural contacts along the anterior parahippocampal gyrus near the entorhinal cortex.

Further studies have validated these initial findings. Vonck and colleagues $(65,66)$ implanted ten patients with amygdalohippocampal electrodes. After a mean follow-up of 31 months (12-52 months): one was seizure free, one experienced $>90 \%$ seizure reduction, five had $>50 \%$ seizure reduction, two had 30 $49 \%$ seizure reduction and one was unchanged. Velasco et $\mathrm{al}^{67}$ reported on nine patients with hippocampal stimulators for at 
least six months. Of the five patients with normal hippocampal imaging, four were seizure free and one nearly so. In the remaining four with hippocampal sclerosis, the average seizure reduction was $70 \%(48-85 \%)$. The benefit in the latter group evolved over a longer period of time than in the non-lesional group.

Tellez-Zenteno et $\mathrm{al}^{68}$ and McLachlan et $\mathrm{al}^{69}$ reported their experience at the University of Western Ontario. A doubleblinded randomized crossover trial involved four patients with unilateral mesial TLE. A longitudinally aligned hippocampal electrode was placed, and patients were randomized to onemonth on- or off-stimulation periods over six months, during which seizure frequency and neuropsychological tests were recorded. There was a median seizure reduction of $15 \%$ with stimulation, but this percentage did not reach significance. A double-blind randomized cross-over study of bilateral hippocampal stimulation in two patients found a $33 \%$ reduction of seizures during the on phase with continued seizure reduction of $25 \%$ during the off phase, returning to baseline in three months.

A randomized trial with large numbers of patients will be needed to determine the effectiveness of hippocampal stimulation in the long term. Controlled Randomized Stimulation Versus Resection (CoRaStiR) (http://clinicaltrials. gov/ct2/show/study/NCT00431457) is a randomized trial of hippocampal stimulation that is recruiting patients. METTLE (A Multicenter Study of Hippocampal Electrical Stimulation in Mesial Temporal Lobe Epilepsy) is a multicenter, parallel-group, double blind randomized controlled trial involving patients with MTLE who may be candidates for resective surgery or whose memory function precludes resective surgery (http://clinical trials.gov/ct2/show/NCT00717431). This study has been terminated due to poor enrolment.

\section{Cortical Stimulation}

Cortical stimulation is commonly used to map function in eloquent brain. This can be done at the time of craniotomy or extraoperatively, when patients have subdural electrodes in place for the investigation of epilepsy. It is known that cortical stimulation can evoke focal after-discharges that may evolve into clinical seizures. It was only a matter of time before cortical stimulation would be investigated as a tool for the treatment of cortical originating seizures.

Lesser et $\mathrm{al}^{70}$ showed that application of brief bursts of $50 \mathrm{~Hz}$ electrical stimulation through subdural electrode contacts could abort stimulation induced after-discharges when carrying out cortical mapping. Yamamoto et $\mathrm{al}^{71}$ reported a patient in whom subdural electrodes had been implanted for investigation of temporal lobe epilepsy. Repeated application of low intensity $(0.5 \mathrm{~mA})$, low frequency $(0.9 \mathrm{~Hz})$ stimulation to an inferior temporal neocortical epileptic focus produced a progressive decrease in interictal spikes. Higher intensity stimulation at the same site (2 mA, 7.5 mA) produced the patient's typical aura and EEG seizure pattern. This bears remarkable similarity to Weiss's quenching phenomenon obtained with low frequency stimulation of the amygdala in kindled rats ${ }^{72}$.

Elisevich et $\mathrm{al}^{73}$ reported a patient with seizures arising from the primary motor area. Chronic cortical stimulation $(50 \mathrm{~Hz}, 450$ $\mathrm{mcsec}, 3 \mathrm{~min} \mathrm{ON}, 10 \mathrm{~min} \mathrm{OFF}$ ) resulted in reduction of seizures from 20-30 events per day a baseline to one event every second day at four years.

\section{Responsive Cortical Stimulation}

The responsive neurostimulation (RNS) device is designed to record cortical electrical activity, detect seizures and deliver stimulation in response to the seizure. Experimental work demonstrating safety and efficacy ${ }^{74,75}$ led the way for the recently completed multicenter double blind randomized controlled trial of $\mathrm{RNS}^{76}$.

In this study 191 patients with one or two seizure foci were enrolled, implanted with depth or surface electrodes and randomized to receive sham or active responsive neurostimulation. The blinded phase entailed four weeks of stimulation optimization then 12 weeks of stimulation delivery. This was followed by an 84 week open-label stimulation phase for all participants.

During the blinded evaluation period there was a $37.9 \%$ reduction in seizures in the treatment group compared to $17.3 \%$ in the sham group. The responder rate (individuals with $\geq 50 \%$ seizure reduction) was $29 \%$ in the treatment group during the blinded evaluation period, $43 \%$ at one year and $46 \%$ at three years. Both sham and treatment groups had similar improvements in secondary outcome measures, including quality of life, at the end of the blinded evaluation period. The treatment group had greater improvements at one and two years into the open-label period in verbal functioning, visuospatial ability, and memory ( $\mathrm{p}<0.05)$.

This study demonstrated the safety and efficacy of RNS. It will be helpful to know whether different areas of the brain were more responsive to this technique than others. Specifics of size of epileptogenic zone, parameters of stimulation, etc will help to refine this technique.

\section{SUMMARY}

There have been significant advances in stimulation of the central nervous system applied to epilepsy over the last couple of decades. A number of similarities arise, regardless of target: there appears to be a latency to beneficial effect, and there appears to be progressive improvement in the degree of seizure reduction in responders over a period of months. Further basic research will help advance the understanding of the underlying mechanisms of these procedures. With all of these techniques, increased numbers and stratification of results to specific epilepsy syndromes will help to allow us to appropriately select patients for these procedures.

The seizure freedom rates with these neuromodulatory techniques do not approach those achieved with resective surgery - leaving them as techniques to be considered in patients who are not resective candidates. 


\section{REFERENCES}

1. Benabid AL, Pollak P, Henry S, de Rougemont J. Combined (thalamotomy and stimulation) stereotactic surgery of the VIM thalamic nucleus for bilateral Parkinson disease. Appl. Neurophysiol. 1987;50(1-6):244-6.

2. Cooper IS, Amin I, Gilman. The effect of chronic cerebellar stimulation upon epilepsy in man. Trans Am Neurol Assoc. 1973;98:192-6.

3. Laxer KD, Robertson LT, Julien RM, Dow RS. Phenytoin: relationship between cerebellar function and epileptic discharges. Adv Neurol. 1980;27:415-27.

4. Krauss GL, Koubeissi MZ. Cerebellar and thalamic stimulation treatment for epilepsy. Acta Neurochir Suppl. 2007;97(Pt. 2):347-56.

5. Wright GD, McLellan DL, Brice JG. A double-blind trial of chronic cerebellar stimulation in twelve patients with severe epilepsy. J Neurol Neurosurg Psychiatry. 1984;47:769-74.

6. Van Buren JM, Wood JH, Oakley J, Hambrecht F. Preliminary evaluation of cerebellar stimulation and biological criteria in the treatment of epilepsy. J Neurosurg. 1978;48:407-16.

7. Davis R, Emmonds SE. Cerebellar stimulation for seizure control: 17-year study. Stereotact Funct Neurosurg. 1992;58:200-8.

8. Cooper IS. Twenty-five years of experience with physiological neurosurgery. Neurosurgery. 1981;9:190-200.

9. Velasco F, Carrillo-Ruiz JD, Brito F, et al. Double-blind, randomized controlled pilot study of bilateral cerebellar stimulation for treatment of intractable motor seizures. Epilepsia. 2005;46(7):1071-81.

10. Bidzinski J, Bacia T, Ostrowski K. Effect of cerebellar cortex electrostimulation on the frequency of seizures in drug-resistant epilepsy. Neurol Neurochir Pol. 1981;31:605-9.

11. Davis R. Cerebellar stimulation for cerebral palsy spasticity, function, and seizures. Arch Med Res. 2000;31:290-9.

12. La GruttaV, Sabatino M, Gravante G, Morici G, Ferraro G, Lagrutta G. A study of caudate inhibition on an epileptic focus in the cat hippocampus. Arch Int Physiol Biochim. 1988;96:113-20.

13. La Grutta V, Amato G, Zagami MT. The importance of the caudate nucleus in the control of convulsive activity in the amygdaloid complex and the temporal cortex of the cat. Electroencephalogr Clin Neurophysiol. 1971;31:57-69.

14. La Grutta V, Sabatino M, Gravante G, La Grutta G. Effects of caudate nucleus on paroxysmal activity in hippocampus of cat. Electroencephalogr. Clin. Neurophysiol. 1985;61:416-21.

15. Psatta DM. Control of chronic experimental focal epilepsy by feedback caudatum stimulations. Epilepsia. 1983;24:444-54.

16. Sorbera F, Crescimanno G, Amato G, La Grutta V. [Effects of conditioning stimulation of the substantia nigra on the epileptic activity of the cat amygdala]. Boll Soc Ital Biol Sper. 1981;57: 1901-4.

17. Chkhenkeli SA, Sramka M, Lortkipanidze GS, et al. Electrophysiological effects and clinical results of direct brain stimulation for intractable epilepsy. Clin Neurol Neurosurg. 2004;106(4):318-29.

18. Chkhenkeli SA, Chkhenkeli IS. Effects of therapeutic stimulation of nucleus caudatus on epileptic electrical activity of brain in patients with intractable epilepsy. Stereotact Funct Neurosurg. 1997;69(1-4 Pt. 2):221-4

19. Sramka M, Fritz G, Galanda M, Nadvornik P. Some observations in treatment stimulation of epilepsy. Acta Neurochir (Wien.) 1976; 23(Suppl.):257-62.

20. Rasmussen T. The neurosurgical treatment of epilepsy. In: Niedermeyer E, editor. Modern Problems of Pharmacopsychiatry. New York: Karger; 1970. p. 306-25.

21. Krauss GL, Fisher RS. Cerebellar and thalamic stimulation for epilepsy. Adv Neurol. 1993;63:231-45.

22. Jasper HH. Current evaluation of the concepts of centrencephalic and cortico-reticular seizures. Electroencephalogr Clin Neurophysiol. 1991;78:2-11.

23. Jasper HH, Droogglever-Furtuyn J. Experimental studies on the functional anatomy of petit mal epilepsy. Assoc Res Nerv Ment Dis. 1947;26:272-98.
24. Williams D. a study of thalamic and cortical rhythms in petit mal. Brain. 1953;76:50-69.

25. Kostopoulos GK. Involvement if the thalamocortical system in epileptic loss of consciousness. Epilepsia. 2001;42(Suppl. 3): 13-9.

26. Hunter $J$, Jasper HH. Effects of thalamic stimulation in unanesthetized animals. The arrest reaction and petit mal like seizure activation patterns and generalized convulsions. Electroencephalogr Clin Neurophysiol. 1949;1:305-24.

27. Starzl TE, Taylor CW, Magoun HW. Ascending conduction in reticular activating system, with special reference to the diencephalon. J Neurophysiol. 1951;14:461-77.

28. Velasco M, Velasco F. State related brain stem regulation of cortical and motor excitability: effects on experimental focal motor seizures. In: Sterman MB, Passouant P, editors. Sleep and Epilepsy. New York: Academic Press; 1982. p. 53-61.

29. Velasco F, Velasco M, Ogarrio C, Fanghanel G. Electrical stimulation of the centromedian thalamic nucleus in the treatment of convulsive seizures: a preliminary report. Epilepsia. 1987;28:421-30.

30. Velasco F. Velasco M, Velasco AL, Jiminez F, Marquez I, Rise M. Electrical stimulation of the centromedian thalamic nucleus in control of seizures: long-term studies. Epilepsia. 1995;26:63-71.

31. Velasco F, Velasco M, Jiminez F, et al. Predictors in the treatment of difficult-to-control seizures by electrical stimulation of the centromedian thalamic nucleus. Neurosurgery. 2000;47: 295-304.

32. Velasco M, Velasco F, Velasco AL. Centromedian-thalamic and hippocampal electrical stimulation for the control of intractable epileptic seizures. J Clin Neurophysiol. 2001;18:495-513.

33. Fisher RS, Uematsu S, Krauss GL, et al. Placebo-controlled pilot study of centromedian thalamic stimulation in treatment of intractable seizures. Epilepsia. 1992;33:841-51.

34. Mirski MA, Ferrendelli JA. Andterio thalamic mediation of generalized pentylenetetrazol seizures. Brain Res. 1986;399: 212-23.

35. Mirski MA, Rossell LA, Terry JB, Fisher RS. Anticonvulsant effect of anterior thalamic high frequency electrical stimulation in the rat. Epilepsy Res. 1997;28:89-100.

36. Mirski MA, Ferrendelli JA. Selective metabolic activation of the mammillary bodies and their connection during ethosuximideinduced suppression of pentylenetetrazol seizures. Epilepsia. 1986;27:194-203.

37. Mirski MA, Fisher RS. Electrical stimulation of the mammillary nuclei increases seizure threshold to pentylenetetrazol in rats. Epilepsia. 1994;35:1309-16.

38. Mirski MA, Ferrendelli JA. Interruption of the mammillothalamic tract prevents seizures in guinea pigs. Science. 1984;226:72-4.

39. Hamani C, Ewerton FI, Bonilha SM, Ballester G, Mello LE, Lozano AM. Bilateral anterior thalamic nucleus lesions and high-frequency stimulation are protective against pilocarpineinduced seizures and status epilepticus. Neurosurgery 2004;54: 191-5.

40. Hamani C, Hodaie M, Chiang J, et al. Deep brain stimulation of the anterior nucleus of the thalamus: effects of electrical stimulation on pilocarpine-induced seizures and status epilepticus. Epilepsy Res. 2008;78(2-3):117-23.

41. Rosenow J, Das K, Rovit RL, Couldwell WT. Irving S. Cooper and his role in intracranial stimulation for movement disorders and epilepsy. Stereotact Funct Neurosurg. 2002;78:95-112.

42. Sussman NM, Goldman HW, Jackel RA, et al. Anterior thalamic stimulation in medically intractable epilepsy. Part II: Preliminary clinical results (abstract). Epilepsia. 1988;29(5):677.

43. Hodaie M, Wennberg RA, Dostrovsky JO, Lozano AM. Chronic anterior thalamus stimulation for intractable epilepsy. Epilepsia. 2002;43(6):603-8.

44. Andrade DM, Zumsteg D, Hamani C, et al. Neurology. 2006;66 (10):1571-3.

45. Fisher R, Salanova V, Witt T, et al. Electrical stimulation of the anterior nucleus of the thalamus for treatment of refractory epilepsy. Epilepsia. 2010;51(5):1528-67. 
46. Iadarola MJ, Gale K. Substantia nigra: site of anticonvulsant activity mediated by gamma-aminobutyric acid. Science. 1982;218:1237-40

47. Garant DS, Gale K. Substantia nigra-mediated anticonvulsant actions: role of nigral output pathways. Exp Neurol. 1987;97: 143-59.

48. Chevalier G, Vacher S, Deniau JM, Desban M. Disinhibition as a basic process in the expression of striatal functions. I. The striato-nigral influence on tecto-spinal/tecto-diencephalic neurons. Brain Res. 1985;334:215-26.

49. Dean P, Gale K. Anticonvulsant action of GABA receptor blockade in the nigrotectal target region. Brain Res. 1989;477:391-95.

50. Redgrave P, Simkins M, Overton P, Dean P. Anticonvulsant role of nigrotectal projection in the maximal electroshock model of epilepsy--I. Mapping of dorsal midbrain with bicuculline. Neuroscience. 1992;46:379-90.

51. Shehab S, Simkins M, Dean P, Redgrave P. Regional distribution of the anticonvulsant and behavioural effects of muscimol injected into the substantia nigra of rats. Eur J Neurosci. 1996;8:749-57.

52. Cavalheiro EA, Turski L. Intrastriatal N-methyl-D-aspartate prevents amygdala kindled seizures in rats. Brain Res. 1986; 377:173-6.

53. Cavalheiro EA, Bortolotto ZA, Turski L. Microinjections of the gamma-aminobutyrate antagonist, bicuculline methiodide, into the caudate-putamen prevent amygdala-kindled seizures in rats. Brain Res. 1987;411:370-2.

54. Deransart C, Le BT, Marescaux C, Depaulis A. Role of the subthalamo-nigral input in the control of amygdala-kindled seizures in the rat. Brain Res. 1998;807(1-2):78-83.

55. Deransart C, Marescaux C, Depaulis A. Involvement of nigral glutamatergic inputs in the control of seizures in a genetic model of absence epilepsy in the rat. Neuroscience. 1996;71:721-8.

56. Vercueil L, Benazzouz A, Deransart C et al. High-frequency stimulation of the subthalamic nucleus suppresses absence seizures in the rat: comparison with neurotoxic lesions. Epilepsy Res. 1998;31:39-46.

57. Usui N, Maesawa S, Kajita Y, Endo O, Takebayashi S, Yoshida J. Suppression of secondary generalization of limbic seizures by stimulation of subthalamic nucleus in rats. J Neurosurg. 2005; 102:1122-9.

58. Lado FA, Velísek L, Moshé SL. The effect of electrical stimulation of the subthalamic nucleus on seizures is frequency dependent. Epilepsia. 2003;44:157-64.

59. Benabid AL, Minotti L, Koudsie A, de Saint MA, Hirsch E. Antiepileptic effect of high-frequency stimulation of the subthalamic nucleus (corpus luysi) in a case of medically intractable epilepsy caused by focal dysplasia: a 30-month follow-up: technical case report. Neurosurgery. 2002;50: 1385-91.

60. Chabardes S, Kahane P, Minotti L, Koudsie A, Hirsch E, Benabid AL. Deep brain stimulation in epilepsy with particular reference to the subthalamic nucleus. Epileptic Disord. 2002;4(Suppl 3): S83-93.

61. Benabid AL, Koudsie A, Chabardes S, et al. Subthalamic nucleus and substantia nigra pars reticularis stimulation: the Grenoble experience. In: Luders HO, editor. Deep Brain Stimulation and Epilepsy. London: Martin Dunitz; 2004. p. 335-48.
62. Neme S, Montgomery EB, Rezai A, Wilson K, Luders HO. Subthalamic nucleus stimulation in patients with intractable epilepsy: the Cleveland experience. In: Luders HO, editor. Deep Brain Stimulation and Epilepsy. London: Martin Dunitz; 2004. p. 349-55.

63. Velasco M, Velasco F, Velasco AL, et al. Subacute electrical stimulation of the hippocampus blocks intractable temporal lobe seizures and paroxysmal EEG activities. Epilepsia 2000;41:15869.

64. Velasco AL, Velasco M, Velasco F, et al. Subacute and chronic electrical stimulation of the hippocampus on intractable temporal lobe seizures: preliminary report. Arch Med Res. 2000; $31: 316-28$.

65. Vonck K, Boon P, Achten E, De Reuck J, Caemaert J. Long-term amygdalohippocampal stimulation for refractory temporal lobe epilepsy. Ann Neurol. 2002;52:556-65.

66. Boon P, Vonck K, De Herdt V, et al. Deep brain stimulation in patients with refractory temporal lobe epilepsy. Epilepsia. 2007; 48:1551-60.

67. Velasco AL, Velasco F, Velasco M, et al. Electrical stimulation of the hippocampal epileptic foci for seizure control: A doubleblind, long-term follow-up study. Epilepsia. 2007;48(10):18951903.

68. Tellez-Zenteno JF, McLachlan RS, Parrent A, Kubu CS, Wiebe S. Hippocampal electrical stimulation in mesial temporal lobe epilepsy. Neurology. 2006;66(10):1490-4.

69. McLachlan RS, Pigott S, Tellez-Zenteno JF, Wiebe S, Parrent A. Bilateral hippocampal stimulation for intractable temporal lobe epilepsy: impact on seizures and memory. Epilepsia. 2010;51(2): 304-7.

70. Lesser RP, Kim SH, Beyderman L, Miglioretti DL, Webber WR, Bare M, Cysyk B, Krauss G, Gordon B. Brief bursts of pulse stimulation terminate afterdischarges caused by cortical stimulation. Neurology. 1999;53:2073-81.

71. Yamamoto J, Ikeda A, Satow T, et al. Low-frequency electric cortical stimulation has an inhibitory effect on epileptic focus in mesial temporal lobe epilepsy. Epilepsia. 2002;43:491-5.

72. Weiss SR, Li XL, Rosen JB, Li H, Heynen T, Post RM. Quenching: inhibition of development and expression of amygdala kindled seizures with low frequency stimulation. Neuroreport. 1995;6: 2171-6.

73. Elisevich K, Jenrow K, Schuh L, Smith B. Long-term electrical stimulation-induced inhibition of partial epilepsy. J Neurosurg. 2006; 105:894-7.

74. Kossoff EH, Ritzl EK, Politsky JM et al. Effect of an external responsive neurostimulator on seizures and electrographic discharges during subdural electrode monitoring. Epilepsia. 2004:45:1560-7.

75. Osorio I, Frei MG, Sunderam S et al. Automated seizure abatement in humans using electrical stimulation. Ann Neurol. 2005;57: 258-68.

76. Morrell MJ and the RNS System in Epilepsy Study Group. Responsive cortical stimulation for the treatment of medically intractable partial epilepsy. Neurology. 2011;77(13):1295-304. 